\title{
Peripheral neuropathy associated with erythrophagocytic lymphohistiocytosis
}

\author{
BERNARD BOUTIN,* MARIE-CLAUDE ROUTON,* FRANCIS ROCCHICCIOLI, $\dagger$ \\ MICHELE MAYER,* GUY LEVERGER, $\ddagger$ OLIVIER ROBAIN,* GERARD PONSOT,* \\ MICHEL ARTHUIS*
}

From the Département de Neurologie Pédiatrique,* Hôpital Saint Vincent de Paul; Institut National de la Santé et de la Recherche Médicale, Unité 188, $\dagger$ Hôpital Saint Vincent de Paul; and Département d'Hématologie

Pédiatrique, $\ddagger$ Hôpital Saint-Louis, France

SUMMARY A 12 year old patient who developed clinical, biochemical and histological features of erythrophagocytic lymphohistiocytosis is described. In contrast to previously reported cases, the prominent neurological feature was a subacute sensorimotor polyneuropathy. Sural nerve biopsy showed a marked reduction of myelinated fibres and severe axonal lesions, absence of histiocyte infiltration and deposits of IgM along the epineurium. In addition to the hypertriglyceridaemia previously described in this condition, an elevation of plasma very long-chain fatty acids and phytanic acid was found which suggests a transient impairment of peroxisomal functions.

Familial erythrophagocytic lymphohistiocytosis is an autosomal recessive disorder characterised by fever, hepatosplenomegaly, pancytopenia and multisystemic infiltration with benign-appearing histiocytes containing erythrocytes. Apart from a few exceptions, ${ }^{12}$ this condition occurs in infancy and is rapidly fatal. Central nervous system (CNS) symptoms have been reported in about $30 \%$ of the cases, ${ }^{34}$ but alterations of the peripheral nervous system were mentioned in only two cases without detailed electrophysiological or histological investigations. ${ }^{12} \mathrm{~A}$ similar lymphohistiocytosis has been subsequently described in association with an active viral infection, and was designated as virus-associated haemophagocytic syndrome (VAHS); it has been reported in children and adults who were previously either healthy or immunosuppressed. ${ }^{56}$

Address for reprint requests: Dr B Boutin, Department de Neurologie Pédiatrique, Hôpital St Vincent de Paul, 74 Av DenfertRochereau, 75674 Paris Cedex 14, France.

Received 26 June 1987. Accepted 8 September 1987

\section{Case report}

A 12 year old girl was referred for the evaluation of a subacute polyneuropathy. She was born to normal nonconsanguineous parents after an uncomplicated pregnancy and delivery, had two normal siblings and no family history of neurological or metabolic disease.

Since her first year, she had had frequent episodes of purulent otitis media and gastroenteritis, which did not interfere with her growth and development rate. She was hospitalised at age 5 years for acute facial paralysis and acute otitis media, both of which resolved within a few weeks; physical examination revealed a marked splenomegaly.

At age 9, she developed fever, asthenia, anorexia, weight loss and began to complain of pain and weakness in the lower extremities. The symptoms worsened rapidly and 2 weeks later she was confined to bed. Physical examination revealed a pale, thin patient with marked splenomegaly, a major muscular atrophy of distal leg muscles, associated with a paresis and vasomotor changes. Laboratory data showed pancytopenia and the bone marrow examination was normal. After several weeks, signs and symptoms resolved spontaneously, but strength did not improve and the child was still unable to walk. Four months later, she relapsed without exacerbation of the neurological symptoms. Examination of the bone marrow revealed hyperplasia with the presence of histiocytes containing erythrocytes. She remitted again. 

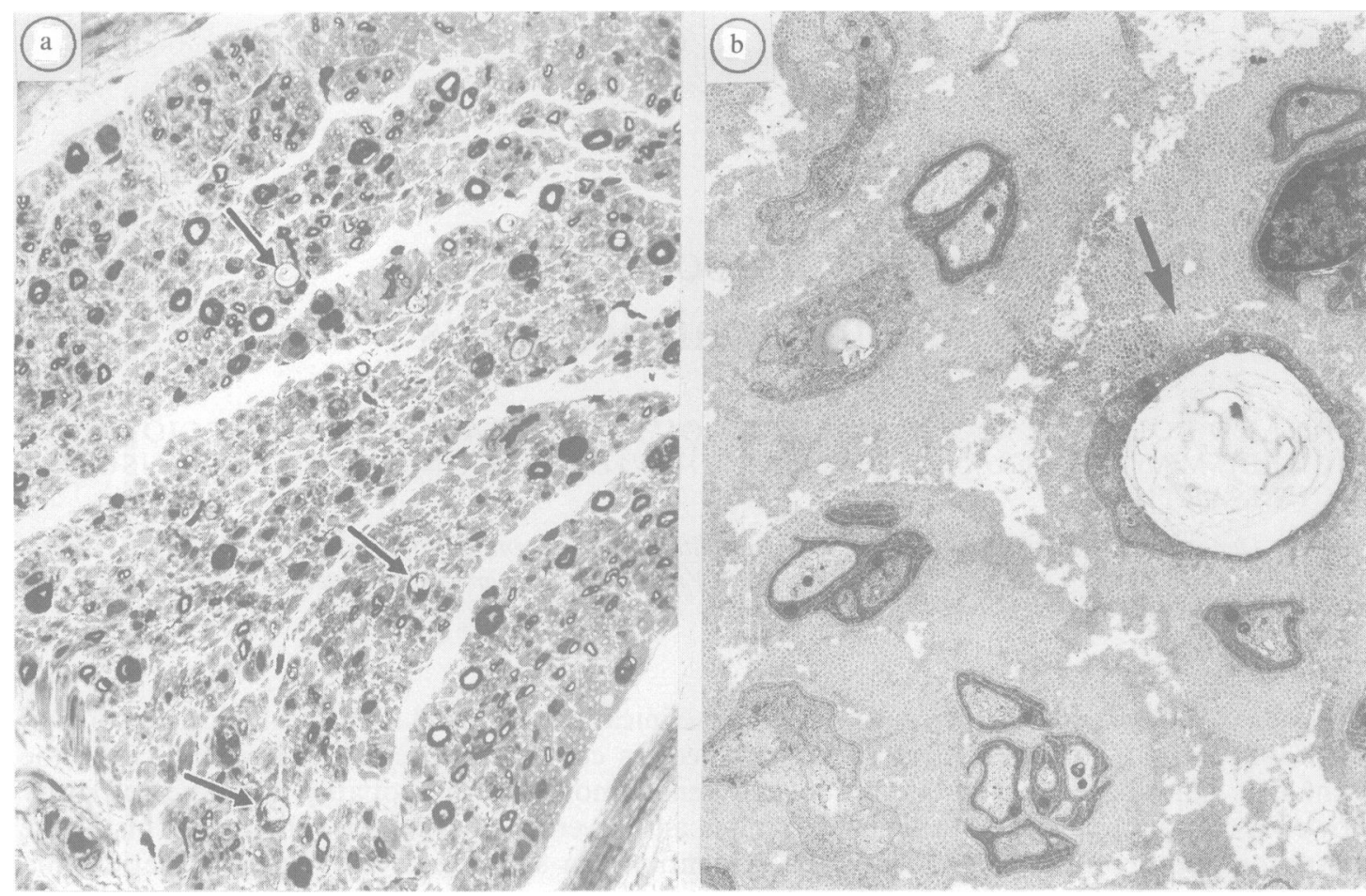

Fig (a) Semithin section of sural nerve, decrease in the density of myelinated fibres, arrows point to some empty sheaths. (Light micrograph $\times 540$.) (b) Sparcity of nerve fibres with proliferation of collagen, arrows point to an empty ring of schwann cytoplasm; note the many single unmyelinated fibres. (Electron micrograph $\times 7400$.)

Six months after the onset of the symptoms, she was referred to our department. On admission, she was pale, thin, afebrile; the spleen was palpable $5 \mathrm{~cm}$ below the left costal margin. There was marked muscular atrophy of the distal leg muscles, strength was rated between 0 and 1 on the MRC scale, tendon reflexes were absent at the knees and ankles, and plantar responses were flexor; sensation to pain, pinprick, touch, vibration and temperature were slightly altered in the feet; there was excessive sweating and marked vasomotor changes; nerves were not palpably thickened, and the rest of the neurological examination was normal. Very rapidly she developed fever, pallor, anorexia, macular rash, hepatosplenomegaly, ascitis, oedema and nuchal rigidity, but the peripheral neuropathy did not worsen.

Laboratory investigations revealed: pancytopenia with a normal bone marrow, hypoproteinaemia $(44 \mathrm{~g} / \mathrm{l})$ with hypo-

Table Very long-chain fatty acids and phytanic acid content in plasma $(u M / l)$

\begin{tabular}{llc}
\hline Plasma $(\mu \mathrm{mol} / \mathrm{l})$ & Patient & \multicolumn{2}{c}{ Controls $(\boldsymbol{n}=30)$} \\
\hline Phytanate & $10 \cdot 5$ & $2 \cdot 05 \pm 0.65$ \\
C22 & $29 \cdot 2$ & $38 \cdot 2 \pm 19.0$ \\
C24 & $37 \cdot 0$ & $27.4 \pm \pm 16 \cdot 2$ \\
C26 & $1 \cdot 50$ & $0 \cdot 35 \pm 0 \cdot 19$ \\
C24/C22 & 1.27 & $0.68 \pm 0.20$ \\
C26/C22 & 0.051 & $0.011 \pm 0.007$ \\
\hline
\end{tabular}

natraemia $(118 \mathrm{mmol} / \mathrm{l})$, coagulation abnormalities witte mainly a fibrinopenia $(0.50 \mathrm{~g} / \mathrm{l})$, hypertriglyceridaemia $(4.8 \Phi$ $\mathrm{mmol} / \mathrm{l}$ ), with normal cholesterol levels and serum lipid electrophoresis; serum bilirubin, liver enzymes and alkaline phosphatases were elevated; analysis of plasma very long chain fatty acids ${ }^{7}$ revealed an elevation of the $\mathrm{C} 26: 0$ fatty acid and phytanic acid, whereas $C 24: 0$ and $C 22: 0$ were normal, as shown in the table. Cerebrospinal fluid (CSF) examination showed 362 lymphocytes, $5.74 \mathrm{~g} / 1$ of protein and 3.1 $\mathrm{mmol} / \mathrm{l}$ of glucose. Serological tests for infectious mononucleosis revealed: the absence of VCA (viral capsid antigen) specific IgM antibodies, the presence of VCA specific IgG antibodies with a titre greater than 1:640, the presence of early antigen with a titre greater than 1:160, the absence of EBNA (Epstein Barr nuclear antigen) antibodies. Immunological results were as follows: the concentrations of serum immunoglobulins were normal, the percentage of $T$ lymphocytes was normal, $\mathbf{T}$ cell subset analysis revealed a decreased T4/T8 ratio; in vitro proliferative responses of lymphocytes were normal to pokeweed mitogen, decreased to phytohaemaglutinin; natural killer cell activity was found to be profoundly impaired and could not be reversed by incubation in vitro with interferon. Chest and skull radiographs and a computed tomographic scan of the head were normal.

She was treated with epipodophyllotoxin 16-213 together with intrathecal methotrexate and steroids, according to the regimen previously described. ${ }^{8}$ Resolution of clinical and 
biochemical abnormalities occurred progressively and resulted in a complete remission except for the neurological signs; despite the treatment, the patient very rapidly developed weakness, paraesthesia and vasomotor changes in the hands. Oral steroids were started $(2 \mathrm{mg} / \mathrm{kg} /$ day) for 15 days, then tapered progressively over 6 weeks. This treatment resulted in a prompt resolution of these signs. No long-term follow up is available.

Electrophysiological and histological studies were performed immediately before the final relapse. Nerve conduction velocity studies and electromyography were normal in the upper limbs; there was marked denervation in the lower limbs, and the motor conduction velocities could not be studied, because of the major distal muscle atrophy. Somato-sensory evoked potentials were normal when the median nerves and absent when the peroneal nerves were stimulated. Visual and auditory evoked responses were normal.

Sural nerve biopsy revealed a marked reduction in the number of myelinated fibres $\left(4200 / \mathrm{mm}^{2}\right)$, the resulting histogram showed a more marked restriction among large myelinated fibres; no onion bulb and no inflammatory cells were found (fig a); teasing was performed on 100 fibres; myelinic alterations were more striking than on transverse sections; no normal large myelinated fibres could be observed; many fibres exhibited linear rows of myelin balls; only small myelinated fibres could be considered normal. Electron microscopic examination revealed alterations of the axons in numerous myelinated fibres, axons being replaced by a slightly electron dense material sometimes exhibiting an irregular lamellar pattern. In a few other myelinated fibres, the lesion was restricted to the myelin sheet with deposits of dark granular material between myelin lamellae. Unmyelinated fibres were restricted in number $\left(20000 / \mathrm{mm}^{2}\right)$, but without major detectable alterations to their structure; many single unmyelinated axons were present; some osmiophilic and clear (presumably) lipid droplets together with accumulation of mitochondria were observed in the Schwann cells (fig b). Transverse sections were examined for the presence of IgG, IgM, IgA, Kappa and lambda chains. Direct immunofluorescence showed positive staining for IgM along the epineurium.

\section{Discussion}

The case reported concerns a child with clinical, biochemical and histological features of erythrophagocytic lymphohistiocytosis. Two forms of this entity have been previously described. The negative family history, the age at onset and the results of serological studies attesting to a recent infection with EBV represent arguments for the diagnosis of virusassociated lymphohistiocytosis. No underlying immune deficiency could be demonstrated: immunological investigations revealed only abnormalities usually present in this disease. ${ }^{9}$ Nevertheless, certain features such as recurrent infections starting in infancy, presence of splenomegaly and a facial palsy at the age of 5 years, are rather in keeping with a protracted perhaps familial lymphohistiocytosis, whose course may have been altered by the occurrence of an EBV infection.

The presence of a subacute polyneuropathy as a prominent neurological symptom was another unusual feature in this patient. There are only two cases of polyneuropathy documented in the literature: one of the three patients from the family reported by Nelson $e t a l^{1}$ and one of the two siblings described by Mozziconacci et $a l^{2}$ who became symptomatic at the ages of 3 and 5 respectively. In our patient, the clinical signs were consistent with a sensorimotor neuropathy, while histological features were suggestive of an axonal degeneration. Different hypotheses can be advanced to explain this neuropathy. Neuropathological reports ${ }^{410}$ have shown that the involvement of the CNS is related to an infiltration by lymphocytes and histiocytes, with eventually multifocal necrosis. Such a mechanism can account for the neuropathy, and the absence of documented infiltration of the nerve may be related either to segmental lesions or to the fact that the biopsy was performed when the patient was in remission. On the other hand we cannot exclude an immunological mechanism: we could not detect inflammatory cells in the biopsy specimen, but the immunostaining for IgM was positive. Such a mechanism could be consistent with the course of the neuropathy and its responsiveness to steroids.

The last particular feature of this case was the presence of biochemical abnormalities which suggest a dysfunction of the peroxysomes. These abnormalities were observed prior to any medication and had disappeared when checked a few weeks after the initiation of treatment. A liver biopsy was performed at that time and revealed the presence of peroxysomes without major alterations. It is difficult to offer a hypothesis concerning the significance of this abnormality in our patient, except that it was not related to any drug and that it could represent another lipid abnormality in erythrophagocytic lymphohistiocytosis. ${ }^{11}$

We thank Dr F Tomé (INSERM, U-153) and Dr J Scotto (INSERM, U-154) for their helpful comments, and Mrs L Sergent for secretarial assistance.

\section{References}

1 Nelson P, Santamaria A, Olson RL, Nayak NC. Generalized lymphohistiocytic infiltration. Pediatrics 1961;27:931-49.

2 Mozziconacci P, Nezelof C, Attal C. La lymphohistiocytose familiale. Arch Fr Pediatr 1965;22:385-92.

3 Perry MC, Harrison EG, Burgert EO, Gilchrist GS. Familial erythrophagocytic lymphohistiocytosis. Cancer 1976;38:209-18.

4 Akima M, Sumi SM. Neuropathology of familial erythrophagocytic lymphohistiocytosis. Hum Pathol 
1984;15:161-8.

5 Risdall RJ, McKenna RW, Nesbit ME, Krivit W. Virusassociated hemophagocytic syndrome: A benign histiocytic proliferation distinct from malignant histiocytosis. Cancer 1979;44:993-1002.

6 Wilson ER, Malluh A, Stagno S, Crist WM. Fatal Epstein-Barr virus-associated hemophagocytic syndrome. J Pediatr 1981;98:260-2.

7 Aubourg P, Bougnères PF, Rocchiccioli F. Capillary gas-liquid chromatographic-mass spectrometric measurement of very long chain $\left(C_{22}\right.$ to $\left.C_{26}\right)$ fatty acids in microliter samples. of plasma. J Lipid Res 1985;26: 263-5.

8 Fischer A, Virelizier JL, Arenzana-Seisdedos F, et al.
Treatment of four patients with erythrophagocytic lymphohistiocytosis by a combination of epipodophyllotoxin, steroids, intrathecal methotrexate and cranial irradiation. Pediatrics 1985;76:263-8.

9 Perez N, Virelizier JL, Arenzana-Seisdedos F, Fischer A. Impaired natural killer activity in lymphohistiocytosis syndrome. J Pediatr 1984;104:569-73.

10 Martin JJ, Cras P. Familial erythrophagocytic lymphohistiocytosis: a neuropathologic study. Acta Neuropathol (Berl) 1985;66:140-4.

11 Ansbacher LE, Singsen BH, Hosler MW, Grimminger H. Familial erythrophagocytic lymphohistiocytosis: an association with serum lipid abnormalities. $J$ Pediatr 1983;102:270-3. 